\title{
On the Approximation of the Derivatives of Spline Quasi-Interpolation in Cubic Spline Space $S_{3}^{1,2}\left(\Delta_{m n}^{(2)}\right)$
}

\author{
Jiang Qian ${ }^{1,2, *}$ and Fan Wang ${ }^{3}$ \\ ${ }^{1}$ College of Sciences, Hohai University, Nanjing 210098, China. \\ ${ }^{2}$ Center for Numerical Simulation Software in Engineering and Sciences, \\ Department of Engineering Mechanics, Hohai University, Nanjing 210098, China. \\ ${ }^{3}$ College of Engineering, Nanjing Agricultural University, Nanjing 210031, China.
}

Received 5 September 2012; Accepted (in revised version) 1 August 2013

Available online 24 January 2014

\begin{abstract}
In this paper, based on the basis composed of two sets of splines with distinct local supports, cubic spline quasi-interpolating operators are reviewed on nonuniform type-2 triangulation. The variation diminishing operator is defined by discrete linear functionals based on a fixed number of triangular mesh-points, which can reproduce any polynomial of nearly best degrees. And by means of the modulus of continuity, the estimation of the operator approximating a real sufficiently smooth function is reviewed as well. Moreover, the derivatives of the nearly optimal variation diminishing operator can approximate that of the real sufficiently smooth function uniformly over quasi-uniform type-2 triangulation. And then the convergence results are worked out.
\end{abstract}

AMS subject classifications: 65D07, 41A25

Key words: Bivariate splines, conformality of smoothing cofactor method, nonuniform type-2 triangulation, quasi-interpolation, modulus of continuity.

\section{Introduction}

As is known, the nonuniform rational $B$-splines scheme has become a de facto standard in Computer Aided Geometric Design, which is a powerful tool for constructing free-form curves and surfaces $[3,7,14,16]$. Due to its rational model, some new alternatives have been proposed for constructing fair-shape-preserving approximations recently $[8-10,15]$. However, both $B$-spline surfaces and the new alternatives are constructed in the form of tensor product, which implies that the degrees of the surfaces are the addition of that of the parameters in two directions so that there may be some inflection points on the surface. Moreover, the bivariate function can not reproduce any polynomial of nearly best degree. Furthermore, it is restricted to construct surfaces over the rectangular mesh. Hence, to

*Corresponding author. Email addresses: qianjianghhu@sina.com (J. Qian), wangfan@njau.edu.cn (F. Wang) 
avoid the shortcomings, it is very important to study multivariate spline functions theoretically. Since multivariate spline functions are heavily dependent on the geometric property of the domain partitions, it is so complex that the non-Cartesian product multivariate spline functions has not been developed radically for a long time. But all is changed until the construction of the Conformality of Smoothing Cofactor Method $[17,18]$.

In a specific way, the smooth cofactor and conformality condition has been introduced to which the polynomials must satisfy by analysing the relation between the polynomials over two adjacent cells $[17,18]$. The conformality condition establishes the equivalent conversion between multivariate spline functions and the corresponding algebraic problems. As a result, the Conformality of Smoothing Cofactor Method provides an algebraic approach to studying the multivariate spline functions, including the dimension and the locally supported basis functions in multivariate spline spaces $[17,18,22,23]$, etc., which are difficult but important. The dimension of the multivariate spline function space $S_{k}^{\mu}(\Delta)$ i.e., the multivariate spline space with degree $k$ and smoothness $\mu$ over the domain $D$ with respect to the partition $\Delta$ have been widely developed in $[4,13,14,17,18,24]$. Recently, Liu, Hong, and Cao [6] determined the dimension and construct a local support basis of the space $S^{1, d}(\Delta(2))$, for $d=0,1$ of the spline functions over the type-2 nonuniform triangulation. The basis functions of bivariate cubic and quartic spline spaces on uniform type- 2 triangulation have been derived in $[5,19]$, respectively, where spline quasi-interpolation has been also investigated thoroughly. These spline quasi-interpolating operators can reproduce any polynomial of (nearly) best degrees, respectively. Moreover, spline quasiinterpolation defined by discrete linear functionals based on a fixed number of triangular mesh-points has been investigated, which showed that they could approximate a real function and its partial derivatives up to an optimal order in $[1,2]$.

However, in view of the complexity in computation of the bases, the study on spline quasi-interpolation over nonuniform type-2 triangulations are almost restricted in bivariate quadratic $B$-splines, see $[20,21]$. Since multivariate approximation over irregular triangulations may be more important than that over uniform triangulations, we have computed the cubic splines in [11], and have constructed the cubic spline quasi-interpolation in [12] by using the Conformality of Smoothing Cofactor Method $[17,18]$. Now we shall make a further study on the approximation of the derivatives of the cubic spline quasi-interpolation in this paper.

A brief outline of this article is organized as follows. In Section 2, we review the dimension and the bases in $S_{3}^{1,2}\left(\Delta_{m n}^{(2)}\right)$. Based on five mesh points or the center of the support of each spline $B_{i j}^{1}$ and five mesh points of the support of each spline $B_{i j}^{2}$, the representation of spline quasi-interpolation is investigated, which can reproduce any polynomial in $\mathbb{P}_{2} \cup\left\{x^{2} y, x y^{2}\right\}$. Then in section 3 , we make a further study of the derivatives of the cubic spline quasi-interpolation, which can approximate the derivatives of the real sufficiently smooth function uniformly over quasi-uniform triangulation.

\section{Review of representation of spline quasi-interpolation in $S_{3}^{1,2}\left(\Delta_{m n}^{(2)}\right)$}

The domain $\Omega=[a, b] \times[c, d]$ is partitioned into $m n$ rectangular cells $\Omega_{i j}=\left[x_{i}, x_{i+1}\right] \times$ $\left[y_{j}, y_{j+1}\right], i=0, \cdots, m-1$ and $j=0, \cdots, n-1$, where $m, n$ are given positive integers, 
and $a=x_{0}<x_{1}<\cdots<x_{m}=b$ and $c=y_{0}<y_{1}<\cdots<y_{n}=d$. Thus, we obtain a type-2 triangulation $\Delta_{m n}^{(2)}$ by adding the two diagonals in each $\Omega_{i j}$. Let $h_{i}=x_{i}-x_{i-1}$, $k_{j}=y_{j}-y_{j-1}$, respectively. In the particular case of $h_{i}=h_{i-1}$ and $k_{j}=k_{j-1}$, such a triangulation is called uniform, otherwise, nonuniform.

A nonuniform bivariate spline $s(x, y) \in S_{3}^{1,2}\left(\Delta_{m n}^{(2)}\right)$ is a piecewise polynomial of degree three satisfying two continuous condition:

(a) $s(x, y)$ is $C^{1}$ continuous on the horizontal and vertical grid segments $x=x_{i}$ and $y=y_{j}$, where $i=0, \cdots, m$ and $j=0, \cdots, n$.

(b) $s(x, y)$ is $C^{2}$ continuous on the diagonal grid segments

$$
y-y_{j}-\frac{k_{j+1}}{h_{i+1}}\left(x-x_{i}\right)=0, \quad y-y_{j}+\frac{k_{j+1}}{h_{i+1}}\left(x-x_{i+1}\right)=0,
$$

where $i=0, \cdots, m-1$ and $j=0, \cdots, n-1$.

The dimension of the nonuniform bivariate spline space $S_{3}^{1,2}\left(\Delta_{m n}^{(2)}\right)$ in [11] is

$$
\operatorname{dim} S_{3}^{1,2}\left(\Delta_{m n}^{(2)}\right)=2 m n+3 m+3 n+4
$$

which is the same with the uniform case in [5].

And also the bases of $S_{3}^{1,2}\left(\Delta_{m n}^{(2)}\right)$ has been calculated in [11] in term of B-net, which is composed of two sets of splines with two kind of distinct supports as shown in Fig. 1 and Fig. 2, respectively. Here, we shall provide the explicit representation of the basis over each triangular cell in the supports as shown in Appendix for the sake of illustration.

Denote by $\Delta_{u}^{1}$ and $\Delta_{v}^{2}$ the triangle cells in the supports, respectively, where the spline functions are expressed in the form of $B_{i j}^{1}\left(\Delta_{u}^{1}\right)(u=1,2, \cdots, 16)$ and $B_{i j}^{2}\left(\Delta_{v}^{2}\right)$ $(v=1,2, \cdots, 24)$, respectively.

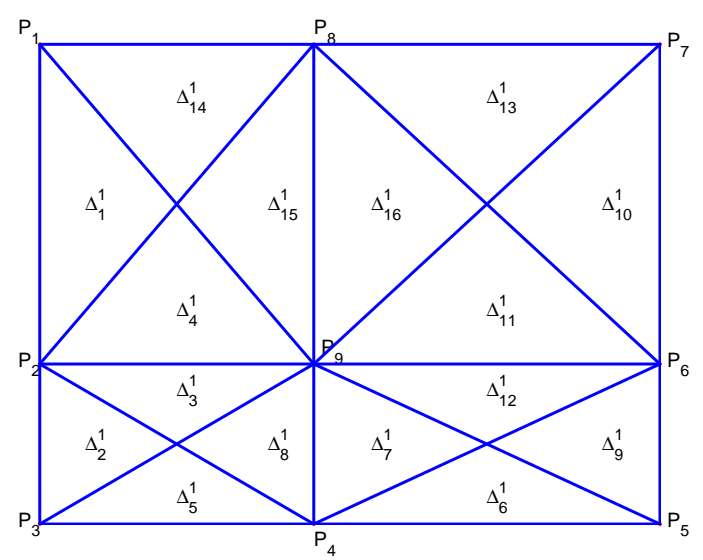

Figure 1: 16 triangular cells in the support of $B_{i j}^{1}(x, y)$. 


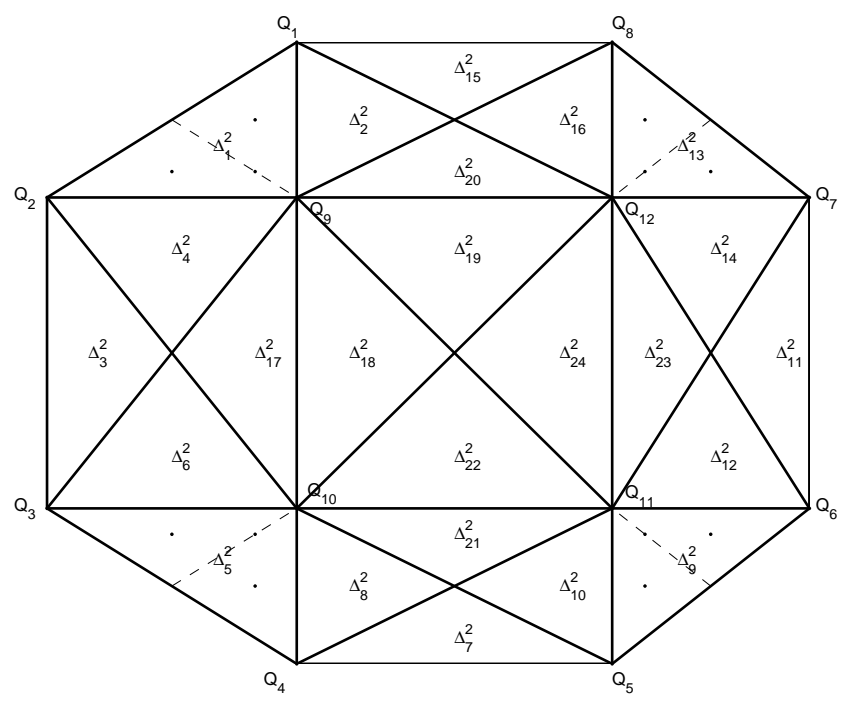

Figure 2: 24 triangular cells in the support of $B_{i j}^{2}(x, y)$.

Let the vertices

$$
\begin{aligned}
& P_{1}\left(x_{i-1}, y_{j+1}\right), \quad P_{2}\left(x_{i-1}, y_{j}\right), \quad P_{3}\left(x_{i-1}, y_{j-1}\right), \quad P_{4}\left(x_{i}, y_{j-1}\right), \quad P_{5}\left(x_{i+1}, y_{j-1}\right), \\
& P_{6}\left(x_{i+1}, y_{j}\right), \quad P_{7}\left(x_{i+1}, y_{j+1}\right), \quad P_{8}\left(x_{i}, y_{j+1}\right), \quad P_{9}\left(x_{i}, y_{j}\right)
\end{aligned}
$$

in the spline $B_{i j}^{1}(x, y)$ with the center $\left(x_{i}, y_{j}\right)$ as shown in Fig. 1 , and the vertices

$$
\begin{array}{llll}
Q_{1}\left(x_{i}, y_{j+2}\right), & Q_{2}\left(x_{i-1}, y_{j+1}\right), & Q_{3}\left(x_{i-1}, y_{j}\right), & Q_{4}\left(x_{i}, y_{j-1}\right), \\
Q_{5}\left(x_{i+1}, y_{j-1}\right), & Q_{6}\left(x_{i+2}, y_{j}\right), & Q_{7}\left(x_{i+2}, y_{j+1}\right), & Q_{8}\left(x_{i+1}, y_{j+2}\right), \\
Q_{9}\left(x_{i}, y_{j+1}\right), & Q_{10}\left(x_{i}, y_{j}\right), & Q_{11}\left(x_{i+1}, y_{j}\right), & Q_{12}\left(x_{i+1}, y_{j+1}\right)
\end{array}
$$

in the spline $B_{i j}^{2}(x, y)$ with the center $\left(\left(x_{i}+x_{i+1}\right) / 2,\left(y_{j}+y_{j+1}\right) / 2\right)$ as shown in Fig. 2.

In view of the translation of the bases, $B_{i j}^{1}(x, y)$ 's and $B_{i j}^{2}(x, y)$ 's do not vanish identically on the domain $\Omega$ defined in Section 2 for the index set

$$
\begin{aligned}
& I_{1}=\{(i, j)=(\alpha, \beta): 0 \leq \alpha \leq m, 0 \leq \beta \leq n\}, \\
& I_{2}=\{(i, j)=(\alpha, \beta):-1 \leq \alpha \leq m,-1 \leq \beta \leq n\},
\end{aligned}
$$

respectively. Then it follows from the cardinality of $I_{1}$ and $I_{2}$ that the total number of the linear independent functions $B_{i j}^{1}(x, y)$ 's and $B_{i j}^{2}(x, y)$ 's which do not vanish identically on the domain $\Omega$ is

$$
2 m n+3 m+3 n+5 \text {. }
$$

By the formula (2.1), it is more than the dimension of $S_{3}^{1,2}\left(\Delta_{m n}^{(2)}\right)$. As a result, we can construct the bases of $S_{3}^{1,2}\left(\Delta_{m n}^{(2)}\right)$ by getting rid of arbitrary one function in either $B_{i j}^{1}(x, y)$ where $(i, j) \in I_{1}$, or $B_{i j}^{2}(x, y)$ where $(i, j) \in I_{2}$. 
Theorem 2.1 (see $[11]$ ). For arbitrary chosen $\left(i_{0}, j_{0}\right) \in I_{1},\left(i_{1}, j_{1}\right) \in I_{2}$, let

$$
\begin{aligned}
& \mathbb{B}^{1}=\left\{B_{i j}^{1}:(i, j) \in I_{1} \backslash\left\{\left(i_{0}, j_{0}\right)\right\}\right\} \bigcup\left\{B_{i j}^{2}:(i, j) \in I_{2}\right\}, \\
& \mathbb{B}^{2}=\left\{B_{i j}^{2}:(i, j) \in I_{2} \backslash\left\{\left(i_{1}, j_{1}\right)\right\}\right\} \bigcup\left\{B_{i j}^{1}:(i, j) \in I_{1}\right\} .
\end{aligned}
$$

Then, either $\mathbb{B}^{1}$ or $\mathbb{B}^{2}$ is a basis of the non-uniform cubic spline space $S_{3}^{1,2}\left(\Delta_{m n}^{(2)}\right)$.

By means of translation of the bases and the values at the ten points on each triangle, it follows that the bases with minimal local support satisfy

Theorem 2.2 (see [11]). For all $(x, y) \in \Omega$

$$
\begin{aligned}
& \sum_{(i, j) \in I_{1}} B_{i j}^{1}(x, y)=1+\lambda, \\
& \sum_{(i, j) \in I_{2}} B_{i j}^{2}(x, y)=-\lambda, \\
& \sum_{(i, j) \in I_{1}} B_{i j}^{1}(x, y)+\sum_{(i, j) \in I_{2}} B_{i j}^{2}(x, y)=1,
\end{aligned}
$$

where $\lambda \neq 0,-1$.

Remark 2.1. With the choice of $\lambda=-0.25$ and -0.75 , the splines $B_{i j}^{1}(x, y)$ 's and $B_{i j}^{2}(x, y)$ 's are shown in Fig. 3 to Fig. 6, respectively.

We have constructed the spline quasi-interpolation by discrete linear functionals based on some mesh-points either in the supports or close to them. These spline quasiinterpolating operators can reproduce polynomials with high degrees, and error estimation show that the variation diminishing operator $V_{m n}(f)$ can approximate a sufficiently smooth function uniformly.

Theorem 2.3 (see [12]). Let $V_{m n}(f)$ be the variation diminishing operator that map $C(\Omega)$ into $S_{3}^{1,2}\left(\Delta_{m n}^{(2)}\right)$ defined by

$$
V_{m n}(f)=\sum_{(i, j) \in I_{1}} \lambda_{i j}(f) B_{i j}^{1}(x, y)+\sum_{(i, j) \in I_{2}} \mu_{i j}(f) B_{i j}^{2}(x, y),
$$

where $\lambda \neq-1,0$, and

$$
\begin{aligned}
\lambda_{i j}(f)= & \frac{1}{3(1+\lambda)} f\left(x_{i}, y_{j}\right), \\
\mu_{i j}(f)=- & \frac{4}{3 \lambda} f\left(\frac{x_{i}+x_{i+1}}{2}, \frac{y_{j}+y_{j+1}}{2}\right)+\frac{1}{6 \lambda}\left[f\left(x_{i}, y_{j+1}\right)+f\left(x_{i+1}, y_{j+1}\right)\right. \\
& \left.+f\left(x_{i}, y_{j}\right)+f\left(x_{i+1}, y_{j}\right)\right] .
\end{aligned}
$$

Then for all $(x, y) \in \Omega$,

$$
V_{m n}(f) \equiv f(x, y), \quad \forall f(x, y) \in \mathbb{P}_{2}[x, y] \cup \operatorname{span}\left\{x^{2} y, x y^{2}\right\} .
$$




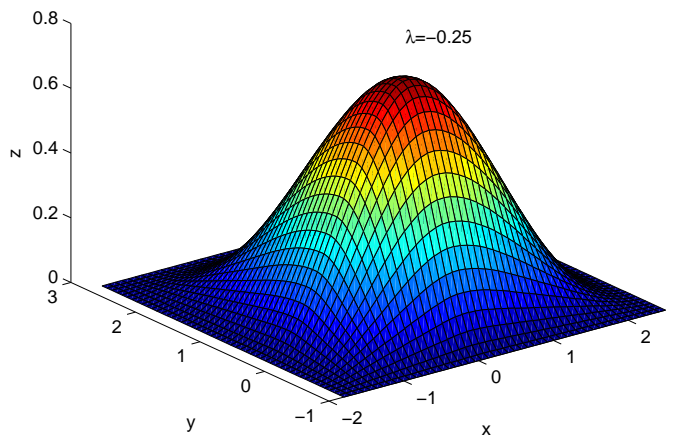

Figure 3: $B_{i j}^{1}(x, y)$ with $\lambda=-0.25$.

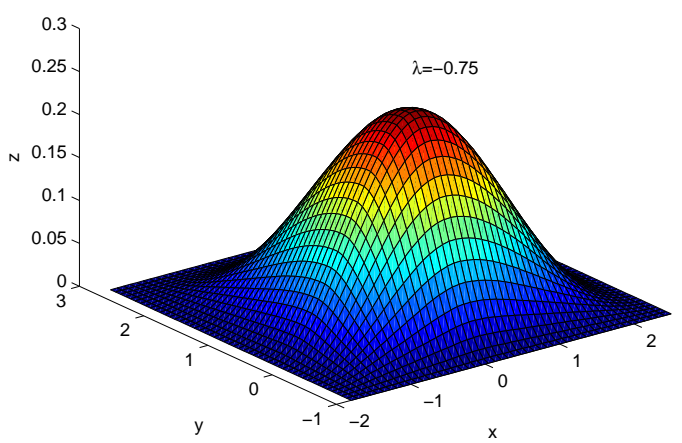

Figure 5: $B_{i j}^{1}(x, y)$ with $\lambda=-0.75$.

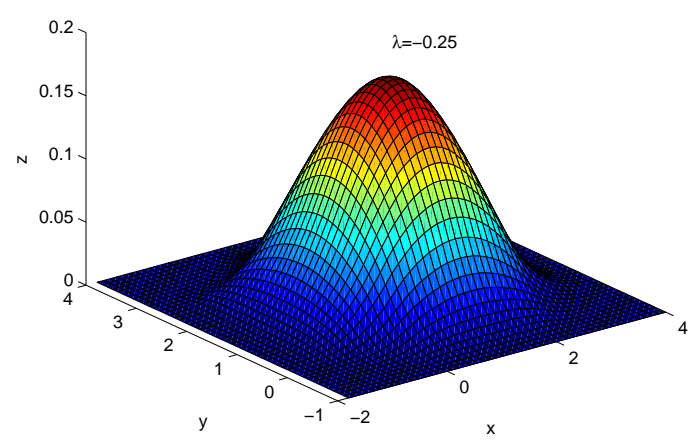

Figure 4: $B_{i j}^{2}(x, y)$ with $\lambda=-0.25$.

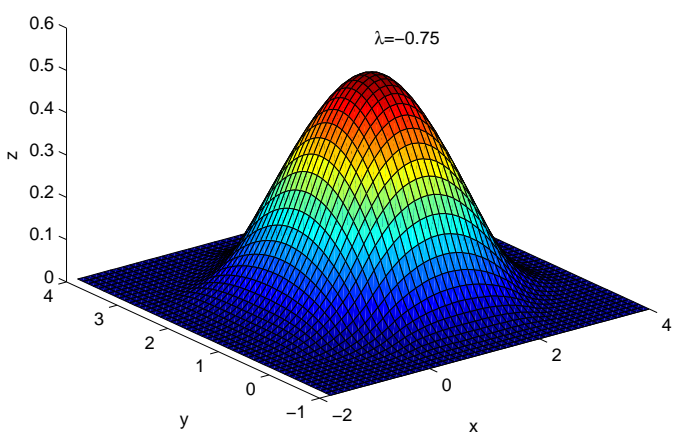

Figure 6: $B_{i j}^{2}(x, y)$ with $\lambda=-0.75$.

Let the compact set $K$ be the closure of the open set containing $\Omega$. The centers of the two distinct supports is located in the interior of $K$ for sufficiently large $m$ and $n$. Let the maximal radius of the two supports of $B_{i j}^{1}$ and $B_{i j}^{2}$ be

$$
\begin{aligned}
& r_{m n, 1}=\max _{0 \leq i \leq m, 0 \leq j \leq n}\left\{\left|P_{9} P_{1}\right|,\left|P_{9} P_{3}\right|,\left|P_{9} P_{5}\right|,\left|P_{9} P_{7}\right|\right\}, \\
& r_{m n, 2}=\max _{-1 \leq i \leq m,-1 \leq j \leq n}\left\{\left|Q Q_{1}\right|,\left|Q Q_{2}\right|,\left|Q Q_{5}\right|,\left|Q Q_{6}\right|\right\},
\end{aligned}
$$

respectively, where $P_{9}\left(x_{i}, y_{j}\right)$ and $Q\left(\left(x_{i}+x_{i+1}\right) / 2,\left(y_{j}+y_{j+1}\right) / 2\right)$ are the centers of the two supports of $B_{i j}^{1}$ and $B_{i j}^{2}$, respectively, and

$$
\begin{array}{rlrl}
\left|P_{9} P_{1}\right| & =\sqrt{h_{i}^{2}+k_{j+1}^{2}}, & \left|P_{9} P_{7}\right|=\sqrt{h_{i+1}^{2}+k_{j+1}^{2}}, \\
\left|P_{9} P_{5}\right|=\sqrt{h_{i+1}^{2}+k_{j}^{2}}, & \left|P_{9} P_{3}\right|=\sqrt{h_{i}^{2}+k_{j}^{2}}, \\
\left|Q Q_{1}\right|=\sqrt{\left(\frac{h_{i+1}}{2}\right)^{2}+\left(k_{j+2}+\frac{k_{j+1}}{2}\right)^{2}}, & \left|Q Q_{2}\right|=\sqrt{\left(h_{i}+\frac{h_{i+1}}{2}\right)^{2}+\left(\frac{k_{j+1}}{2}\right)^{2}}, \\
\left|Q Q_{5}\right|=\sqrt{\left(\frac{h_{i+1}}{2}\right)^{2}+\left(k_{j}+\frac{k_{j+1}}{2}\right)^{2}}, & \left|Q Q_{6}\right|=\sqrt{\left(h_{i+2}+\frac{h_{i+1}}{2}\right)^{2}+\left(\frac{k_{j+1}}{2}\right)^{2}} .
\end{array}
$$


Denote by

$$
\begin{array}{rlrl}
h_{m n} & =\max _{-1 \leq i \leq m+2}\left\{h_{i}\right\}, & k_{m n} & =\max _{-1 \leq j \leq n+2}\left\{h_{j}\right\}, \\
\delta_{m n} & =\max \left\{h_{m n}, k_{m n}\right\}, & \delta_{m n}^{*}=\max \left\{r_{m n, 1}, r_{m n, 2}\right\},
\end{array}
$$

and

$$
\begin{aligned}
& \omega^{k}(f)=\max _{l=0, \cdots, k}\left\{\omega_{\Omega}\left(\frac{\partial^{k} f}{\partial^{k-l} x \partial^{l} y}, \frac{\delta_{m n}}{2}\right)\right\}, \\
& \left\|D^{k} f\right\|=\max _{l=0, \cdots, k} \sup _{(x, y) \in \Omega}\left\{\left|\frac{\partial^{k} f}{\partial^{k-l} x \partial^{l} y}\right|\right\},
\end{aligned}
$$

where $\omega_{\Omega}$ is defined as the modulus of continuity, and $k=1,2, \cdots$. Let $\|\cdot\|_{\Omega}$ be the supremum over $\Omega$, and we shall work out the estimation.

Theorem 2.4 (see [12]). Let $f \in C(K)$, for sufficiently large positive zeals $m$ and $n$,

$$
\left\|f-V_{m n}(f)\right\|_{\Omega} \leq \frac{7}{3} \omega_{\Omega}\left(f, \delta_{m n}^{*}\right) .
$$

When $f \in C^{1}(\Omega)$, then

$$
\left\|f-V_{m n}(f)\right\|_{\Omega} \leq \frac{10}{3} \delta_{m n} \omega^{1}(f) .
$$

When $f \in C^{2}(\Omega)$, then

$$
\left\|f-V_{m n}(f)\right\|_{\Omega} \leq \frac{5}{3} \delta_{m n}^{2} \omega^{2}(f) .
$$

When $f \in C^{3}(\Omega)$, then

$$
\left\|f-V_{m n}(f)\right\|_{\Omega} \leq \frac{5}{9} \delta_{m n}^{3}\left\|D^{3} f\right\| .
$$

\section{The approximation of the derivatives of the spline quasi-interpolating operator}

In this section, we shall make further study of the nearly optimal cubic spline quasiinterpolating operator $V_{m n}(f)$, which indicates that its derivatives can approximate the derivatives of a smooth function uniformly based on the modulus of continuity. In much detail, we consider three cases as follows.

Case one: we shall consider for $f \in C^{1}$,

$$
E_{s t 1}(\tilde{x}, \tilde{y})=D^{s t} V_{m n}(f)(\tilde{x}, \tilde{y}), \quad s+t=1,
$$

where $(\tilde{x}, \tilde{y}) \in \Omega$ and $D^{s t}=\partial^{s+t} / \partial^{s} x \partial^{t} y$.

By using the reproduction of $V_{m n}(f)$, and the Taylor representation of $f$ at the point $(\tilde{x}, \tilde{y}) \in \Omega$

$$
f(x, y)=f(\tilde{x}, \tilde{y})+f_{x}^{\prime}\left(u_{1}, v_{1}\right)(x-\tilde{x})+f_{y}^{\prime}\left(u_{1}, v_{1}\right)(y-\tilde{y}),
$$


where

$$
\left(u_{1}, v_{1}\right)=\epsilon_{1}(\tilde{x}, \tilde{y})+\left(1-\epsilon_{1}\right)(x, y), \quad \epsilon_{1} \in(0,1)
$$

we have

$$
\begin{aligned}
& \left|D^{s t}\left(V_{m n}(f)\right)\right|=\left|D^{s t} V_{m n}(f-f(\tilde{x}, \tilde{y}))\right| \\
\leq & \sum_{(i, j) \in I_{1}}\left|\lambda_{i j}(f-f(\tilde{x}, \tilde{y}))\right|\left|D^{s t} B_{i j}^{1}\right|+\sum_{(i, j) \in I_{2}}\left|\mu_{i j}(f-f(\tilde{x}, \tilde{y}))\right|\left|D^{s t} B_{i j}^{2}\right| .
\end{aligned}
$$

Thus it is sufficient to derive the boundary of $\mid \lambda_{i j}\left(f-f(\tilde{x}, \tilde{y})|,| \mu_{i j}(f-f(\tilde{x}, \tilde{y})) \mid\right.$, $\left|D^{s t} B_{i j}^{1}\right|,\left|D^{s t} B_{i j}^{2}\right|$. In fact, analogous to the proof of Theorem 2.4, it follows

$$
\begin{aligned}
& \left|\lambda_{i j}(f-f(\tilde{x}, \tilde{y}))\right| \leq \frac{2}{3|1+\lambda|} \delta_{m n}\left\|D^{1} f\right\|, \\
& \left|\mu_{i j}(f-f(\tilde{x}, \tilde{y}))\right| \leq \frac{2}{|\lambda|} \delta_{m n}\left\|D^{1} f\right\| .
\end{aligned}
$$

Moreover, by computing directly, one can obtain the partial derivatives of the two sets of splines $B_{i j}^{1}$ and $B_{i j}^{2}$ which depend on the values at three vertices and three midpoints on three edges over each triangular cell as shown in Figs. 7 and 8, respectively. The values of the partial derivatives $D^{10} B_{i j}^{1}$ and $D^{10} B_{i j}^{2}$ are listed in Tables 1 and 2, respectively, where

$$
A_{i}=\frac{h_{i}}{h_{i}+h_{i+1}}, \quad A_{i}^{\prime}=\frac{h_{i+1}}{h_{i}+h_{i+1}}, \quad B_{j}=\frac{k_{j}}{k_{j}+k_{j+1}}, \quad B_{j}^{\prime}=\frac{k_{j+1}}{k_{j}+k_{j+1}},
$$

and $\forall \lambda(\neq 0,-1) \in \mathbb{R}$ throughout this paper. And the values of $D^{01} B_{i j}^{1}$ and $D^{01} B_{i j}^{2}$ can be calculated as well, which are omitted here. It should be noted that all the values of $D^{s t} B_{i j}^{1}$

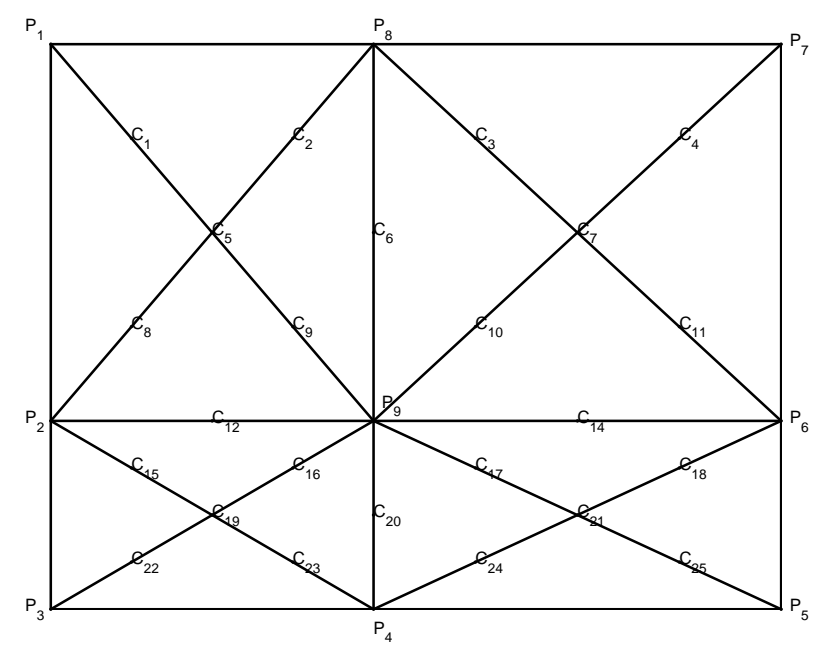

Figure 7: Middle points on the edges in the support of $B_{i j}^{1}$. 


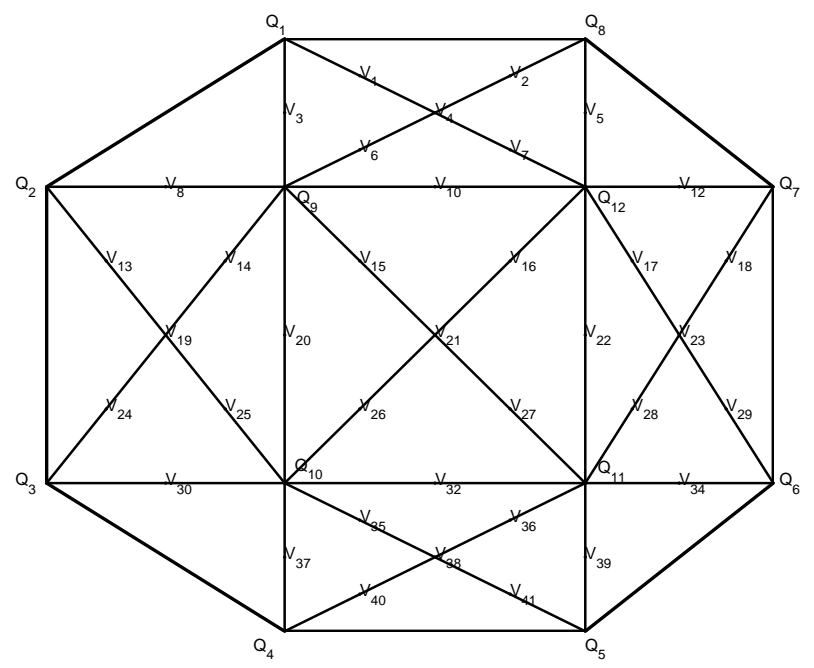

Figure 8: Middle points on the edges in the support of $B_{i j}^{2}$.

and $D^{s t} B_{i j}^{2}$ are equal to zero on the boundary of the supports for $s+t=1$. After resolving the maximal values of $D^{s t} B_{i j}^{1}$ and $D^{s t} B_{i j}^{2}$ on each triangular cell, we have

Theorem 3.1. Let $(x, y)$ in any triangular cell in the supports of $B_{i j}^{1}$ and the supports of $B_{i j}^{2}$. Then

$$
\left|D^{s t} B_{i j}^{1}\right| \leq \frac{a_{1}|1+\lambda|}{\tilde{h}_{i}^{s} \tilde{k}_{j}^{t}}, \quad\left|D^{s t} B_{i j}^{2}\right| \leq \frac{b_{1}|\lambda|}{\tilde{h}_{i}^{s} \tilde{k}_{j}^{t}},
$$

where $s+t=1, \tilde{h_{i}}=\min \left\{h_{i}, h_{i+1}, h_{i+2}\right\}, \tilde{k_{j}}=\min \left\{k_{j}, k_{j+1}, k_{j+2}\right\}$, and $a_{1}, b_{1}$ are real constant.

Hence, by means of (3.4) and (3.5), it follows

Theorem 3.2. For $f(x, y) \in C^{1},(x, y) \in \Omega$,

$$
\left|D^{s t}\left(V_{m n}(f)\right)\right| \leq\left(\frac{2 a_{1}}{3}+4 b_{1}\right) \frac{\delta_{m n}}{\tilde{\delta}_{m n}}\left\|D^{1} f\right\|,
$$

where $\tilde{\delta}_{m n}=\min _{i j}\left\{\tilde{h}_{i}, \tilde{k}_{j}\right\}$.

Case two: we shall consider for $f \in C^{2}$,

$$
E_{s t 2}(\tilde{x}, \tilde{y})= \begin{cases}D^{s t}\left(f-V_{m n}(f)\right)(\tilde{x}, \tilde{y}), & s+t=1, \\ D^{s t} V_{m n}(f)(\tilde{x}, \tilde{y}), & s+t=2 .\end{cases}
$$

When $s+t=1$, by using the reproduction of $V_{m n}(f)$, Theorem 3.1, and the Taylor representation of $f(3.2)$, that is,

$$
\begin{gathered}
f(x, y)=q_{1}(x, y)+\left[f_{x}^{\prime}\left(u_{1}, v_{1}\right)-f_{x}^{\prime}(x, y)\right](x-\tilde{x}) \\
+\left[f_{y}^{\prime}\left(u_{1}, v_{1}\right)-f_{y}^{\prime}(x, y)\right](y-\tilde{y}),
\end{gathered}
$$


Table 1: The values of $D^{10} B_{i j}^{1}(x, y)(\lambda \neq-1,0)$ in the support.

\begin{tabular}{||cccc||}
\hline Points & Values & Points & Values \\
\hline$C_{1}$ & $\frac{3(1+\lambda)}{16 h_{i}}$ & $C_{14}$ & $-\frac{3(1+\lambda)}{2 h_{i+1}}$ \\
$C_{2}$ & $\frac{3(1+\lambda)}{16 h_{i}}$ & $C_{15}$ & $\frac{15(1+\lambda)}{16 h_{i}}$ \\
$C_{3}$ & $-\frac{3(1+\lambda)}{16 h_{i+1}}$ & $C_{16}$ & $\frac{15(1+\lambda)}{16 h_{i}}$ \\
$C_{4}$ & $-\frac{3(1+\lambda)}{16 h_{i+1}}$ & $C_{17}$ & $-\frac{15(1+\lambda)}{16 h_{i+1}}$ \\
$C_{5}$ & $\frac{3(1+\lambda)}{4 h_{i}}$ & $C_{18}$ & $-\frac{15(1+\lambda)}{16 h_{i+1}}$ \\
$C_{6}$ & 0 & $C_{19}$ & $\frac{3(1+\lambda)}{4 h_{i}}$ \\
$C_{7}$ & $-\frac{3(1+\lambda)}{4 h_{i+1}}$ & $C_{20}$ & 0 \\
$C_{8}$ & $\frac{15(1+\lambda)}{16 h_{i}}$ & $C_{21}$ & $-\frac{3(1+\lambda)}{4 h_{i+1}}$ \\
$C_{9}$ & $\frac{15(1+\lambda)}{16 h_{i}}$ & $C_{22}$ & $\frac{3(1+\lambda)}{16 h_{i}}$ \\
$C_{10}$ & $-\frac{15(1+\lambda)}{16 h_{i+1}}$ & $C_{23}$ & $\frac{3(1+\lambda)}{16 h_{i}}$ \\
$C_{11}$ & $-\frac{15(1+\lambda)}{16 h_{i+1}}$ & $C_{24}$ & $-\frac{3(1+\lambda)}{16 h_{i+1}}$ \\
$C_{12}$ & $\frac{3(1+\lambda)}{2 h_{i}}$ & $C_{25}$ & $-\frac{3(1+\lambda)}{16 h_{i+1}}$ \\
$P_{9}$ & 0 & & \\
\hline
\end{tabular}

we have

$$
\begin{aligned}
& \left|D^{s t}\left(f-V_{m n}(f)\right)\right|=\left|D^{s t} V_{m n}\left(f-q_{1}\right)\right| \\
\leq & \sum_{(i, j) \in I_{1}}\left|\lambda_{i j}\left(f-q_{1}\right)\right|\left|D^{s t} B_{i j}^{1}\right|+\sum_{(i, j) \in I_{2}}\left|\mu_{i j}\left(f-q_{1}\right)\right|\left|D^{s t} B_{i j}^{2}\right| \\
\leq & \left(\frac{2 a_{1}}{3}+4 b_{1}\right) \frac{\delta_{m n}}{\tilde{\delta}_{m n}}\left\|\tilde{\omega}^{1} f\right\|,
\end{aligned}
$$

where

$$
\tilde{\omega}^{k}(f)=\max _{l=0, \cdots, k}\left\{\omega_{\Omega}\left(\frac{\partial^{k} f}{\partial^{k-l} x \partial^{l} y}, \delta_{m n}\right)\right\}, \quad k=1,2, \cdots .
$$

When $s+t=2$, by using the reproduction of $V_{m n}(f)$, and the Taylor representation of $f$ at the point $(\tilde{x}, \tilde{y}) \in \Omega$

$$
f(x, y)=q_{1}(x, y)+\frac{1}{2}\left[(x-\tilde{x}) \frac{\partial}{\partial x}+(y-\tilde{y}) \frac{\partial}{\partial y}\right]^{2} f\left(u_{2}, v_{2}\right)
$$

where

$$
q_{1}(x, y)=f(\tilde{x}, \tilde{y})+f_{x}^{\prime}(\tilde{x}, \tilde{y})(x-\tilde{x})+f_{y}^{\prime}(\tilde{x}, \tilde{y})(y-\tilde{y})
$$

and

$$
\left(u_{2}, v_{2}\right)=\epsilon_{2}(x, y)+\left(1-\epsilon_{2}\right)(\tilde{x}, \tilde{y}), \quad \epsilon_{2} \in(0,1)
$$


Table 2: The values of $D^{10} B_{i j}^{2}(x, y)(\lambda \neq-1,0)$ in the support.

\begin{tabular}{||clcl||}
\hline Points & Values & Points & Values \\
\hline$V_{1}$ & 0 & $V_{22}$ & $\frac{\lambda}{h_{i+2}} A_{i+1}^{\prime}\left[\frac{27}{16}-\frac{3}{4}\left(B_{j}^{\prime}+B_{j+1}\right)\right]$ \\
$V_{2}$ & 0 & $V_{23}$ & $\frac{3 \lambda}{4 h_{i+2}} A_{i+1}^{\prime}$ \\
$V_{3}$ & $-\frac{3 \lambda}{4 h_{i}} A_{i} B_{j+1}^{\prime}$ & $V_{24}$ & $-\frac{3 \lambda}{16 h_{i}} A_{i}$ \\
$V_{4}$ & 0 & $V_{25}$ & $\frac{\lambda}{h_{i}} A_{i}\left(-\frac{27}{16}+\frac{3}{4} B_{j}^{\prime}\right)$ \\
$V_{5}$ & $\frac{3 \lambda}{4 h_{i+2}} A_{i+1}^{\prime} B_{j+1}^{\prime}$ & $V_{26}$ & $\frac{3 \lambda}{16 h_{i+1}} A_{i+1}-\frac{\lambda}{h_{i+1}} A_{i}^{\prime}\left(\frac{15}{16}+\frac{3}{4} B_{j}\right)$ \\
$V_{6}$ & $-\frac{3 \lambda}{4 h_{i+1}} A_{i}^{\prime} B_{j+1}^{\prime}$ & $V_{27}$ & $-\frac{3 \lambda}{16 h_{i+1}} A_{i}^{\prime}+\frac{\lambda}{h_{i+1}} A_{i+1}\left(\frac{15}{16}+\frac{3}{4} B_{j}\right)$ \\
$V_{7}$ & $\frac{3 \lambda}{4 h_{i+1}} A_{i+1} B_{j+1}^{\prime}$ & $V_{28}$ & $\frac{\lambda}{h_{i+2}} A_{i+1}^{\prime}\left(\frac{27}{16}-\frac{3}{4} B_{j}^{\prime}\right)$ \\
$V_{8}$ & $-\frac{3 \lambda}{4 h_{i}} A_{i} B_{j+1}^{\prime}$ & $V_{29}$ & $\frac{3 \lambda}{16 h_{i+2}} A_{i+1}^{\prime}$ \\
$Q_{9}$ & $-\frac{3 \lambda}{h_{i}} A_{i} B_{j+1}^{\prime}$ & $V_{30}$ & $-\frac{3 \lambda}{4 h_{i}} A_{i} B_{j}$ \\
$V_{10}$ & $\frac{3 \lambda}{4 h_{i+1}}\left(A_{i+1}-A_{i}^{\prime}\right) B_{j+1}^{\prime}$ & $Q_{10}$ & $-\frac{3 \lambda}{h_{i}} A_{i} B_{j}$ \\
$Q_{12}$ & $\frac{3 \lambda}{h_{i+2}} A_{i+1}^{\prime} B_{j+1}^{\prime}$ & $V_{32}$ & $\frac{3 \lambda}{4 h_{i+1}}\left(A_{i+1}-A_{i}^{\prime}\right) B_{j}$ \\
$V_{12}$ & $\frac{3 \lambda}{4 h_{i+2}} A_{i+1}^{\prime} B_{j+1}^{\prime}$ & $Q_{11}$ & $\frac{3 \lambda}{h_{i+1}} A_{i+1} B_{j}$ \\
$V_{13}$ & $-\frac{3 \lambda}{16 h_{i}} A_{i}$ & $V_{34}$ & $\frac{3 \lambda}{4 h_{i+2}} A_{i+1}^{\prime} B_{j}$ \\
$V_{14}$ & $\frac{\lambda}{h_{i}} A_{i}\left(-\frac{27}{16}+\frac{3}{4} B_{j+1}\right)$ & $V_{35}$ & $-\frac{3 \lambda}{4 h_{i+1}} A_{i}^{\prime} B_{j}$ \\
$V_{15}$ & $\frac{3 \lambda}{16 h_{i+1}} A_{i+1}-\frac{\lambda}{h_{i+1}} A_{i}^{\prime}\left(\frac{15}{16}+\frac{3}{4} B_{j+1}^{\prime}\right)$ & $V_{36}$ & $\frac{3 \lambda}{4 h_{i+1}} A_{i+1} B_{j}$ \\
$V_{16}$ & $-\frac{3 \lambda}{16 h_{i+1}} A_{i}^{\prime}+\frac{\lambda}{h_{i+1}} A_{i+1}\left(\frac{15}{16}+\frac{3}{4} B_{j+1}^{\prime}\right)$ & $V_{37}$ & $-\frac{3 \lambda}{4 h_{i}} A_{i} B_{j}$ \\
$V_{17}$ & $\frac{\lambda}{h_{i+2}} A_{i+1}^{\prime}\left(\frac{27}{16}-\frac{3}{4} B_{j+1}\right)$ & $V_{38}$ & 0 \\
$V_{18}$ & $\frac{3 \lambda}{16 h_{i+2}} A_{i+1}^{\prime}$ & $V_{39}$ & $\frac{3 \lambda}{4 h_{i+2}} A_{i+1}^{\prime} B_{j}$ \\
$V_{19}$ & $-\frac{3 \lambda}{4 h_{i}} A_{i}$ & $V_{40}$ & 0 \\
$V_{20}$ & $\frac{\lambda}{h_{i}} A_{i}\left[-\frac{27}{16}+\frac{3}{4}\left(B_{j}^{\prime}+B_{j+1}\right)\right]$ & $V_{41}$ & 0 \\
$V_{21}$ & $\frac{3 \lambda}{4 h_{i+1}}\left(A_{i+1}-A_{i}^{\prime}\right)$ & & \\
\hline
\end{tabular}

we have

$$
\begin{aligned}
& \left|D^{s t}\left(V_{m n}(f)\right)\right|=\left|D^{s t} V_{m n}\left(f-q_{1}\right)\right| \\
\leq & \sum_{(i, j) \in I_{1}}\left|\lambda_{i j}\left(f-q_{1}\right)\right|\left|D^{s t} B_{i j}^{1}\right|+\sum_{(i, j) \in I_{2}}\left|\mu_{i j}\left(f-q_{1}\right)\right|\left|D^{s t} B_{i j}^{2}\right| .
\end{aligned}
$$

Analogous to Case one, we have

$$
\begin{aligned}
& \left|\lambda_{i j}\left(f-q_{1}\right)\right| \leq \frac{2}{3|1+\lambda|} \delta_{m n}^{2}\left\|D^{1} f\right\|, \\
& \left|\mu_{i j}(f-f(\tilde{x}, \tilde{y}))\right| \leq \frac{4}{|\lambda|} \delta_{m n}^{2}\left\|D^{1} f\right\| .
\end{aligned}
$$


Moreover, as $D^{s t} B_{i j}^{1}$ and $D^{s t} B_{i j}^{2}$ are linear polynomial in each triangular cell in $\Omega$ for $s+t=$ 2 , the maximal values on the triangular cell depend on their values at the three vertices, respectively. After simple computation, it follows:

Theorem 3.3. Let $(x, y)$ in any triangular cell in the supports of $B_{i j}^{1}$ and the supports of $B_{i j}^{2}$. Then for $s+t=2$

$$
\left|D^{s t} B_{i j}^{1}\right| \leq \frac{a_{2}|1+\lambda|}{\tilde{h}_{i}^{s} \tilde{k}_{j}^{t}}, \quad\left|D^{s t} B_{i j}^{2}\right| \leq \frac{b_{2}|\lambda|}{\tilde{h}_{i}^{s} \tilde{k}_{j}^{t}},
$$

where $a_{2}, b_{2}$ are real constant.

Hence, by means of (3.14) and (3.15), it follows

$$
\begin{aligned}
& \left|D^{s t}\left(V_{m n}(f)\right)\right|=\left|D^{s t} V_{m n}\left(f-q_{1}\right)\right| \\
\leq & \sum_{(i, j) \in I_{1}}\left|\lambda_{i j}\left(f-q_{1}\right)\right|\left|D^{s t} B_{i j}^{1}\right|+\sum_{(i, j) \in I_{2}}\left|\mu_{i j}\left(f-q_{1}\right)\right|\left|D^{s t} B_{i j}^{2}\right| \\
\leq & \left(\frac{2 a_{2}}{3}+4 b_{2}\right) \frac{\delta_{m n}^{2}}{\tilde{\delta}_{m n}^{2}}\left\|D^{2} f\right\| .
\end{aligned}
$$

As a result, it follows

Theorem 3.4. For $f(x, y) \in C^{2},(\tilde{x}, \tilde{y}) \in \Omega$,

$$
\begin{array}{ll}
\mid D^{s t}\left(\left(f-V_{m n}(f)\right) \mid \leq\left(\frac{2 a_{1}}{3}+4 b_{1}\right) \frac{\delta_{m n}}{\tilde{\delta}_{m n}}\left\|\tilde{\omega}^{1} f\right\|,\right. & s+t=1, \\
\left|D^{s t}\left(V_{m n}(f)\right)\right| \leq\left(\frac{2 a_{2}}{3}+4 b_{2}\right) \frac{\delta_{m n}^{2}}{\tilde{\delta}_{m n}^{2}}\left\|D^{2} f\right\|, & s+t=2 .
\end{array}
$$

Case three: we shall consider for $f \in C^{3}$,

$$
E_{s t 2}(\tilde{x}, \tilde{y})= \begin{cases}D^{s t}\left(f-V_{m n}(f)\right)(\tilde{x}, \tilde{y}), & s+t=1,2, \\ D^{s t} V_{m n}(f)(\tilde{x}, \tilde{y}), & s+t=3 .\end{cases}
$$

When $s+t=1$, by means of the reproduction of $V_{m n}(f)$, Theorem 3.1, and the Taylor representation of $f$ at the point $(\tilde{x}, \tilde{y}) \in \Omega$

$$
\begin{aligned}
f(x, y)= & q_{2}(x, y)+\frac{1}{2}\left\{\left[f_{x x}^{\prime \prime}\left(u_{2}, v_{2}\right)-f_{x x}^{\prime \prime}(\tilde{x}, \tilde{y})\right](x-\tilde{x})^{2}+2\left[f_{x y}^{\prime \prime}\left(u_{2}, v_{2}\right)\right.\right. \\
& \left.\left.-f_{x y}^{\prime \prime}(\tilde{x}, \tilde{y})\right] \cdot(x-\tilde{x})(y-\tilde{y})+\left[f_{y y}^{\prime \prime}\left(u_{2}, v_{2}\right)-f_{y y}^{\prime \prime}(\tilde{x}, \tilde{y})\right](y-\tilde{y})^{2}\right\},
\end{aligned}
$$

where

$$
q_{2}(x, y)=q_{1}(x, y)+\frac{1}{2}\left[(x-\tilde{x}) \frac{\partial}{\partial x}+(y-\tilde{y}) \frac{\partial}{\partial y}\right]^{2} f\left(u_{2}, v_{2}\right)
$$

and

$$
\left(u_{2}, v_{2}\right)=\epsilon_{2}(x, y)+\left(1-\epsilon_{2}\right)(\tilde{x}, \tilde{y}), \quad \epsilon_{2} \in(0,1),
$$


it follows

$$
\begin{aligned}
& \left|D^{s t}\left(f-V_{m n}(f)\right)\right|=\left|D^{s t} V_{m n}\left(f-q_{2}\right)\right| \\
\leq & \sum_{(i, j) \in I_{1}}\left|\lambda_{i j}\left(f-q_{2}\right)\right|\left|D^{s t} B_{i j}^{1}\right|+\sum_{(i, j) \in I_{2}}\left|\mu_{i j}\left(f-q_{2}\right)\right|\left|D^{s t} B_{i j}^{2}\right| \\
\leq & \left(\frac{2 a_{1}}{3}+4 b_{1}\right) \frac{\delta_{m n}^{2}}{\tilde{\delta}_{m n}} \tilde{\omega}^{2} f,
\end{aligned}
$$

where $\tilde{\omega}^{2} f$ is defined in (3.10). It should be noted that the formula

$$
D^{s t}\left(f-V_{m n}(f)\right)=D^{s t}\left(f-q_{2}-V_{m n}\left(f-q_{2}\right)\right)=-D^{s t} V_{m n}\left(f-q_{2}\right)
$$

at $(\tilde{x}, \tilde{y}) \in \Omega$ holds for $s+t=0,1,2$, for the formula $f-q_{2}$ is equal to 0 at the point $(\tilde{x}, \tilde{y})$, by means of the Taylor representation

$$
f(x, y)=q_{2}(x, y)+\frac{1}{3 !}\left[(x-\tilde{x}) \frac{\partial}{\partial x}+(y-\tilde{y}) \frac{\partial}{\partial y}\right]^{3} f\left(u_{3}, v_{3}\right),
$$

where

$$
\left(u_{3}, v_{3}\right)=\epsilon_{3}(x, y)+\left(1-\epsilon_{3}\right)(\tilde{x}, \tilde{y}), \quad \epsilon_{3} \in(0,1) .
$$

When $s+t=2$, analogous to the case of $s+t=1$, it follows

$$
\begin{aligned}
& \left|D^{s t}\left(f-V_{m n}(f)\right)\right|=\left|D^{s t} V_{m n}\left(f-q_{2}\right)\right| \\
\leq & \sum_{(i, j) \in I_{1}}\left|\lambda_{i j}\left(f-q_{2}\right)\right|\left|D^{s t} B_{i j}^{1}\right|+\sum_{(i, j) \in I_{2}}\left|\mu_{i j}\left(f-q_{2}\right)\right|\left|D^{s t} B_{i j}^{2}\right| \\
\leq & \left(\frac{2 a_{1}}{3}+4 b_{1}\right) \frac{\delta_{m n}^{2}}{\tilde{\delta}_{m n}^{2}} \tilde{\omega}^{2} f .
\end{aligned}
$$

When $s+t=3$, the values of $D^{s t} B_{i j}^{1}$ and $D^{s t} B_{i j}^{2}$ are constant on each triangular cell in $\Omega$, respectively. We would like to make a list of the values of $D^{30} B_{i j}^{1}$ and $D^{21} B_{i j}^{1}$ on each triangular cell in the support in Table 3 , while $D^{30} B_{i j}^{2}$ and $D^{21} B_{i j}^{2}$ in Tables 4 and 5. It is apparent that one can obtain the values of $D^{03} B_{i j}^{1}, D^{03} B_{i j}^{2}, D^{12} B_{i j}^{1}$ and $D^{12} B_{i j}^{2}$ on each triangular cell in the supports, respectively, which are omitted here. Thus we have

Theorem 3.5. Let $(x, y)$ in any triangular cell in the supports of $B_{i j}^{1}$ and the supports of $B_{i j}^{2}$. Then for $s+t=3$

$$
\left|D^{s t} B_{i j}^{1}\right| \leq \frac{a_{3}|1+\lambda|}{\tilde{h}_{i}^{s} \tilde{k}_{j}^{t}}, \quad\left|D^{s t} B_{i j}^{2}\right| \leq \frac{b_{3}|\lambda|}{\tilde{h}_{i}^{s} \tilde{k}_{j}^{t}},
$$

where $a_{3}, b_{3}$ are real constant. 
Table 3: The values of $D^{30} B_{i j}^{1}(x, y)$ and $D^{21} B_{i j}^{1}(x, y)$ on triangular cells in the support.

\begin{tabular}{||cll||}
\hline Triangular cells & $D^{30} B_{i j}^{1}(x, y)$ & $D^{21} B_{i j}^{1}(x, y)$ \\
\hline$\Delta_{1}^{1}$ & $-\frac{6(1+\lambda)}{h_{i}^{3}}$ & $-\frac{6(1+\lambda)}{h_{i}^{2} k_{j+1}}$ \\
$\Delta_{2}^{1}$ & $-\frac{6(1+\lambda)}{h_{i}^{3}}$ & $\frac{6(1+\lambda)}{h_{i}^{2} k_{j}}$ \\
$\Delta_{3}^{1}$ & $-\frac{12(1+\lambda)}{h_{i}^{3}}$ & 0 \\
$\Delta_{4}^{1}$ & $-\frac{12(1+\lambda)}{h_{i}^{3}}$ & 0 \\
$\Delta_{5}^{1}$ & 0 & 0 \\
$\Delta_{6}^{1}$ & 0 & 0 \\
$\Delta_{7}^{1}$ & $\frac{6(1+\lambda)}{h_{i+1}^{3}}$ & $-\frac{6(1+\lambda)}{h_{i+1}^{2} k_{j}}$ \\
$\Delta_{8}^{1}$ & $-\frac{6(1+\lambda)}{h_{i}^{3}}$ & $-\frac{6(1+\lambda)}{h_{i}^{2} k_{j}}$ \\
$\Delta_{9}^{1}$ & $\frac{6(1+\lambda)}{h_{i+1}^{3}}$ & $\frac{6(1+\lambda)}{h_{i+1}^{2} k_{j}}$ \\
$\Delta_{10}^{1}$ & $\frac{6(1+\lambda)}{h_{i+1}^{3}}$ & $-\frac{6(1+\lambda)}{h_{i+1}^{2} k_{j+1}}$ \\
$\Delta_{11}^{1}$ & $\frac{12(1+\lambda)}{h_{i+1}^{3}}$ & 0 \\
$\Delta_{12}^{1}$ & $\frac{12(1+\lambda)}{h_{i+1}^{3}}$ & 0 \\
$\Delta_{13}^{1}$ & 0 & 0 \\
$\Delta_{14}^{1}$ & 0 & 0 \\
$\Delta_{15}^{1}$ & $-\frac{6(1+\lambda)}{h_{i}^{3}}$ & $\frac{6(1+\lambda)}{h_{i}^{2} k_{j+1}}$ \\
$\Delta_{16}^{1}$ & $\frac{6(1+\lambda)}{h_{i+1}^{3}}$ & $\frac{6(1+\lambda)}{h_{i+1}^{2} k_{j+1}}$ \\
\hline & &
\end{tabular}
have

By Theorem 3.5, the reproduction of $V_{m n}(f)$, and the Taylor representation (3.22), we

$$
\begin{aligned}
& \left|D^{s t} V_{m n}(f)\right|=\left|D^{s t} V_{m n}\left(f-q_{2}\right)\right| \\
\leq & \sum_{(i, j) \in I_{1}}\left|\lambda_{i j}\left(f-q_{2}\right)\right|\left|D^{s t} B_{i j}^{1}\right|+\sum_{(i, j) \in I_{2}}\left|\mu_{i j}\left(f-q_{2}\right)\right|\left|D^{s t} B_{i j}^{2}\right| \\
\leq & \frac{4}{3}\left(\frac{a_{3}}{3}+2 b_{3}\right) \frac{\delta_{m n}^{3}}{\tilde{\delta}_{m n}^{3}}\left\|D^{3} f\right\| .
\end{aligned}
$$

As a result, it follows

Theorem 3.6. For $f(x, y) \in C^{3},(\tilde{x}, \tilde{y}) \in \Omega$,

$$
\begin{array}{ll}
\mid D^{s t}\left(\left(f-V_{m n}(f)\right) \mid \leq\left(\frac{2 a_{1}}{3}+4 b_{1}\right) \frac{\delta_{m n}^{2}}{\tilde{\delta}_{m n}} \tilde{\omega}^{2} f,\right. & s+t=1, \\
\mid D^{s t}\left(\left(f-V_{m n}(f)\right) \mid \leq\left(\frac{2 a_{2}}{3}+4 b_{2}\right) \frac{\delta_{m n}^{2}}{\tilde{\delta}_{m n}^{2}} \tilde{\omega}^{2} f,\right. & s+t=2,
\end{array}
$$


Table 4: The values of $D^{30} B_{i j}^{2}(x, y)$ and $D^{21} B_{i j}^{2}(x, y)$ on triangular cells in the support (Part I).

\begin{tabular}{||cll||}
\hline Triangular cells & $D^{30} B_{i j}^{2}(x, y)$ & $D^{21} B_{i j}^{2}(x, y)$ \\
\hline$\Delta_{1}^{2}$ & $-\frac{6 \lambda}{h_{i}^{3}} A_{i} B_{j+1}^{\prime}$ & $\frac{6 \lambda}{h_{i}^{2} k_{j+2}} A_{i} B_{j+1}^{\prime}$ \\
$\Delta_{2}^{2}$ & $-\frac{6 \lambda}{h_{i+1}^{3}} A_{i}^{\prime} B_{j+1}^{\prime}$ & $-\frac{6 \lambda}{h_{i+1}^{2} k_{j+2}} A_{i}^{\prime} B_{j+1}^{\prime}$ \\
$\Delta_{3}^{2}$ & $-\frac{6 \lambda}{h_{i}^{3}} A_{i}$ & 0 \\
$\Delta_{4}^{2}$ & $-\frac{6 \lambda}{h_{i}^{3}} A_{i} B_{j+1}^{\prime}$ & $\frac{6 \lambda}{h_{i}^{2} k_{j+1}} A_{i} B_{j+1}$ \\
$\Delta_{5}^{2}$ & $-\frac{6 \lambda}{h_{i}^{3}} A_{i} B_{j}$ & $-\frac{6 \lambda}{h_{i}^{2} k_{j}} A_{i} B_{j}$ \\
$\Delta_{6}^{2}$ & $-\frac{6 \lambda}{h_{i}^{3}} A_{i} B_{j}$ & $-\frac{6 \lambda}{h_{i}^{2} k_{j+1}} A_{i} B_{j}^{\prime}$ \\
$\Delta_{7}^{2}$ & 0 & 0 \\
$\Delta_{8}^{2}$ & $-\frac{6 \lambda}{h_{i+1}^{3}} A_{i}^{\prime} B_{j}$ & $\frac{6 \lambda}{h_{i+1}^{2} k_{j}} A_{i}^{\prime} B_{j}$ \\
$\Delta_{9}^{2}$ & $\frac{6 \lambda}{h_{i+2}^{3}} A_{i+1}^{\prime} B_{j}$ & $-\frac{6 \lambda}{h_{i+2}^{2} k_{j}} A_{i+1}^{\prime} B_{j}$ \\
$\Delta_{10}^{2}$ & $\frac{6 \lambda}{h_{i+1}^{3}} A_{i+1} B_{j}$ & $\frac{6 \lambda}{h_{i+1}^{2} k_{j}} A_{i+1} B_{j}$ \\
$\Delta_{11}^{2}$ & $\frac{6 \lambda}{h_{i+2}^{3}} A_{i+1}^{\prime}$ & 0 \\
$\Delta_{12}^{2}$ & $\frac{6 \lambda}{h_{i+2}^{3}} A_{i+1}^{\prime} B_{j}$ & $-\frac{6 \lambda}{h_{i+2}^{2} k_{j+1}} A_{i+1}^{\prime} B_{j}^{\prime}$ \\
$\Delta_{13}^{2}$ & $\frac{6 \lambda}{h_{i+2}^{3}} A_{i+1}^{\prime} B_{j+1}^{\prime}$ & $\frac{6 \lambda}{h_{i+2}^{2} k_{j+2}} A_{i+1}^{\prime} B_{j+1}^{\prime}$ \\
$\Delta_{14}^{2}$ & $\frac{6 \lambda}{h_{i+2}^{3}} A_{i+1}^{\prime} B_{j+1}^{\prime}$ & $\frac{6 \lambda}{h_{i+2}^{2} k_{j+1}} A_{i+1}^{\prime} B_{j+1}$ \\
$\Delta_{15}^{2}$ & 0 & 0 \\
$\Delta_{16}^{2}$ & $\frac{6 \lambda}{h_{i+1}^{3}} A_{i+1} B_{j+1}^{\prime}$ & $-\frac{6 \lambda}{h_{i+1}^{2} k_{j+2}} A_{i+1} B_{j+1}^{\prime}$ \\
$\Delta_{17}^{2}$ & $\frac{6 \lambda}{h_{i}^{3}} A_{i}\left(B_{j+1}-B_{j}\right)$ & $\frac{6 \lambda}{h_{i}^{2} k_{j+1}} A_{i}\left(B_{j+1}-B_{j}^{\prime}\right)$ \\
$\Delta_{18}^{2}$ & $\frac{6 \lambda}{h_{i+1}^{3}}\left[A_{i}^{\prime}\left(B_{j+1}-B_{j}\right)+A_{i+1}\right]$ & $\frac{6 \lambda}{h_{i+1}^{2} k_{j+1}} A_{i}^{\prime}\left(B_{j}^{\prime}-B_{j+1}\right)$ \\
\hline & & \\
& & \\
& & \\
& &
\end{tabular}

Table 5: The values of $D^{30} B_{i j}^{2}(x, y)$ and $D^{21} B_{i j}^{2}(x, y)$ on triangular cells in the support (Part II).

\begin{tabular}{||cll||}
\hline Triangular cells & $D^{30} B_{i j}^{2}(x, y)$ & $D^{21} B_{i j}^{2}(x, y)$ \\
\hline$\Delta_{19}^{2}$ & $\frac{6 \lambda}{h_{i+1}^{3}}\left(A_{i+1}-A_{i}^{\prime}\right) B_{j+1}^{\prime}$ & $-\frac{6 \lambda}{h_{i+1}^{2} k_{j+1}}\left(A_{i}^{\prime}+A_{i+1}\right) B_{j+1}$ \\
$\Delta_{20}^{2}$ & $\frac{6 \lambda}{h_{i+1}^{3}}\left(A_{i+1}-A_{i}^{\prime}\right) B_{j+1}^{\prime}$ & $-\frac{6 \lambda}{h_{i+1}^{2} k_{j+2}}\left(A_{i}^{\prime}+A_{i+1}\right) B_{j+1}^{\prime}$ \\
$\Delta_{21}^{2}$ & $\frac{6 \lambda}{h_{i+1}^{3}}\left(A_{i+1}-A_{i}^{\prime}\right) B_{j}$ & $\frac{6 \lambda}{h_{i+1}^{2} k_{j}}\left(A_{i}^{\prime}+A_{i+1}\right) B_{j}$ \\
$\Delta_{22}^{2}$ & $\frac{6 \lambda}{h_{i+1}^{3}}\left(A_{i+1}-A_{i}^{\prime}\right) B_{j}$ & $\frac{6 \lambda}{h_{i+1}^{2} k_{j+1}}\left(A_{i}^{\prime}+A_{i+1}\right) B_{j}^{\prime}$ \\
$\Delta_{23}^{2}$ & $\frac{6 \lambda}{h_{i+2}^{3}} A_{i+1}^{\prime}\left(B_{j+1}^{\prime}-B_{j+1}^{\prime}\right)$ & $\frac{6 \lambda}{h_{i+2}^{2} k_{j+1}} A_{i+1}^{\prime}\left(B_{j+1}-B_{j}^{\prime}\right)$ \\
$\Delta_{24}^{2}$ & $\frac{6 \lambda}{h_{i+1}^{3}}\left[A_{i+1}\left(B_{j}-B_{j+1}\right)-A_{i}^{\prime}\right]$ & $\frac{6 \lambda}{h_{i+1}^{2} k_{j+1}} A_{i+1}\left(B_{j}^{\prime}-B_{j+1}\right)$ \\
\hline
\end{tabular}




$$
\left|D^{s t}\left(V_{m n}(f)\right)\right| \leq\left(\frac{4 a_{3}}{9}+\frac{8 b_{3}}{3}\right) \frac{\delta_{m n}^{3}}{\tilde{\delta}_{m n}^{3}}\left\|D^{3} f\right\|, \quad s+t=3 .
$$

By investigating the theorems in above, one can conclude that the item $\delta_{m n} / \tilde{\delta}_{m n}$ plays a role in the uniform approximation of the derivatives. Thus we shall recall the following definition introduced in [2]. Similarly,

Definition 3.1. A sequence of type-2 triangulation $\Delta_{m n}^{(2)}$ of $\Omega$ is quasi-uniform if there exists a positive constant $\rho$ such that

$$
\frac{h_{m n}}{\tilde{h}_{m n}} \leq \rho, \quad \frac{h_{m n}}{\tilde{k}_{m n}} \leq \rho, \quad \frac{k_{m n}}{\tilde{h}_{m n}} \leq \rho, \quad \frac{k_{m n}}{\tilde{h}_{m n}} \leq \rho .
$$

Moreover a sequence of cubic spline space $S_{3}^{1,2}\left(\Delta_{m n}^{(2)}\right)$ is quasi-uniform if they are based on a sequence of quasi-uniform type-2 triangulation.

Therefore, we can conclude

Remark 3.1. $D^{s t} V_{m n}(f)$ approximates $D^{s t} f$ uniformly as $\delta_{m n}$ approaches to 0 for $f \in$ $C^{r-1}(\Omega), 1<r \leq s+t \leq 3$ over quasi-uniform type-2 uniform. In fact, by Definition 3.1, it follows that $\left(\delta_{m n} / \tilde{\delta}_{m n}\right)^{s+t}$ are bounded.

Acknowledgments The authors wish to express our great appreciation to Prof. Renhong Wang for his valuable suggestions. Also, the authors would like to thank Dr. Chongjun Li and Dr. Chungang Zhu for their help.

This work is supported by National Basic Research Program of China (973 Project No. 2010CB832702), R and D Special Fund for Public Welfare Industry (Hydrodynamics, Grant No. 201101014), National Science Funds for Distinguished Young Scholars (Grant No. 11125208) and Programme of Introducing Talents of Discipline to Universities (111 project, Grant No. B12032).

This work is supported by the Fundamental Research Funds for the Central Universities, and Hohai University Postdoctoral Science Foundation 2016-412051.

\section{Appendix}

The representation of the splines $B_{i j}^{1}(x, y), B_{i j}^{2}(x, y)$ on each triangular cell in the supports is established as follows where $\lambda \in(-1,0)$.

$$
\begin{aligned}
& B_{i j}^{1}\left(\Delta_{1}^{1}\right)=(1+\lambda)\left[-\frac{1}{h_{i}^{3}}\left(x-x_{i-1}\right)-\frac{3}{h_{i}^{2} k_{j+1}}\left(y-y_{j+1}\right)\right]\left(x-x_{i-1}\right)^{2}, \\
& B_{i j}^{1}\left(\Delta_{2}^{1}\right)=(1+\lambda)\left[-\frac{1}{h_{i}^{3}}\left(x-x_{i-1}\right)+\frac{3}{h_{i}^{2} k_{j}}\left(y-y_{j-1}\right)\right]\left(x-x_{i-1}\right)^{2},
\end{aligned}
$$




$$
\begin{aligned}
& B_{i j}^{1}\left(\Delta_{3}^{1}\right)=(1+\lambda)\left[-\frac{1}{h_{i}^{3}}\left(x-x_{i-1}\right)+\frac{3}{h_{i}^{2} k_{j}}\left(y-y_{j-1}\right)\right]\left(x-x_{i-1}\right)^{2} \\
& -\frac{1+\lambda}{k_{j}^{3}}\left[y-y_{j}+\frac{k_{j}}{h_{i}}\left(x-x_{i-1}\right)\right]^{3} \text {, } \\
& B_{i j}^{1}\left(\Delta_{4}^{1}\right)=(1+\lambda)\left[-\frac{1}{h_{i}^{3}}\left(x-x_{i-1}\right)-\frac{3}{h_{i}^{2} k_{j+1}}\left(y-y_{j+1}\right)\right]\left(x-x_{i-1}\right)^{2} \\
& +\frac{1+\lambda}{k_{j+1}^{3}}\left[y-y_{j}-\frac{k_{j+1}}{h_{i}}\left(x-x_{i-1}\right)\right]^{3} \text {, } \\
& B_{i j}^{1}\left(\Delta_{5}^{1}\right)=(1+\lambda)\left[\frac{3}{h_{i} k_{j}^{2}}\left(x-x_{i-1}\right)-\frac{1}{k_{j}^{3}}\left(y-y_{j-1}\right)\right]\left(y-y_{j-1}\right)^{2}, \\
& B_{i j}^{1}\left(\Delta_{6}^{1}\right)=(1+\lambda)\left[-\frac{3}{h_{i+1} k_{j}^{2}}\left(x-x_{i+1}\right)-\frac{1}{k_{j}^{3}}\left(y-y_{j-1}\right)\right]\left(y-y_{j-1}\right)^{2} \text {, } \\
& B_{i j}^{1}\left(\Delta_{7}^{1}\right)=(1+\lambda)\left[-\frac{3}{h_{i+1} k_{j}^{2}}\left(x-x_{i+1}\right)-\frac{1}{k_{j}^{3}}\left(y-y_{j-1}\right)\right]\left(y-y_{j-1}\right)^{2} \\
& -\frac{1+\lambda}{k_{j}^{3}}\left[y-y_{j-1}-\frac{k_{j}}{h_{i+1}}\left(x-x_{i}\right)\right]^{3} \text {, } \\
& B_{i j}^{1}\left(\Delta_{8}^{1}\right)=(1+\lambda)\left[\frac{3}{h_{i} k_{j}^{2}}\left(x-x_{i-1}\right)-\frac{1}{k_{j}^{3}}\left(y-y_{j-1}\right)\right]\left(y-y_{j-1}\right)^{2} \\
& -\frac{1+\lambda}{k_{j}^{3}}\left[y-y_{j-1}+\frac{k_{j}}{h_{i}}\left(x-x_{i}\right)\right]^{3} \text {, } \\
& B_{i j}^{1}\left(\Delta_{9}^{1}\right)=(1+\lambda)\left[\frac{1}{h_{i+1}^{3}}\left(x-x_{i+1}\right)+\frac{3}{h_{i+1}^{2} k_{j}}\left(y-y_{j-1}\right)\right]\left(x-x_{i+1}\right)^{2} \text {, } \\
& B_{i j}^{1}\left(\Delta_{10}^{1}\right)=(1+\lambda)\left[\frac{1}{h_{i+1}^{3}}\left(x-x_{i+1}\right)-\frac{3}{h_{i+1}^{2} k_{j+1}}\left(y-y_{j+1}\right)\right]\left(x-x_{i+1}\right)^{2} \text {, } \\
& B_{i j}^{1}\left(\Delta_{11}^{1}\right)=(1+\lambda)\left[\frac{1}{h_{i+1}^{3}}\left(x-x_{i+1}\right)-\frac{3}{h_{i+1}^{2} k_{j+1}}\left(y-y_{j+1}\right)\right]\left(x-x_{i+1}\right)^{2} \\
& +\frac{1+\lambda}{k_{j+1}^{3}}\left[y-y_{j}+\frac{k_{j+1}}{h_{i+1}}\left(x-x_{i+1}\right)\right]^{3} \text {, } \\
& B_{i j}^{1}\left(\Delta_{12}^{1}\right)=(1+\lambda)\left[\frac{1}{h_{i+1}^{3}}\left(x-x_{i+1}\right)+\frac{3}{h_{i+1}^{2} k_{j}}\left(y-y_{j-1}\right)\right]\left(x-x_{i+1}\right)^{2} \\
& -\frac{1+\lambda}{k_{j}^{3}}\left[y-y_{j}-\frac{k_{j}}{h_{i+1}}\left(x-x_{i+1}\right)\right]^{3} \text {, } \\
& B_{i j}^{1}\left(\Delta_{13}^{1}\right)=(1+\lambda)\left[-\frac{3}{h_{i+1} k_{j+1}^{2}}\left(x-x_{i+1}\right)+\frac{1}{k_{j+1}^{3}}\left(y-y_{j+1}\right)\right]\left(y-y_{j+1}\right)^{2} \text {, }
\end{aligned}
$$




$$
\begin{aligned}
& B_{i j}^{1}\left(\Delta_{14}^{1}\right)=(1+\lambda)\left[\frac{3}{h_{i} k_{j+1}^{2}}\left(x-x_{i-1}\right)+\frac{1}{k_{j+1}^{3}}\left(y-y_{j+1}\right)\right]\left(y-y_{j+1}\right)^{2}, \\
& B_{i j}^{1}\left(\Delta_{15}^{1}\right)=(1+\lambda)\left[\frac{3}{h_{i} k_{j+1}^{2}}\left(x-x_{i-1}\right)+\frac{1}{k_{j+1}^{3}}\left(y-y_{j+1}\right)\right]\left(y-y_{j+1}\right)^{2} \\
& +\frac{1+\lambda}{k_{j+1}^{3}}\left[y-y_{j+1}-\frac{k_{j+1}}{h_{i}}\left(x-x_{i}\right)\right]^{3} \text {, } \\
& B_{i j}^{1}\left(\Delta_{16}^{1}\right)=(1+\lambda)\left[-\frac{3}{h_{i+1} k_{j+1}^{2}}\left(x-x_{i+1}\right)+\frac{1}{k_{j+1}^{3}}\left(y-y_{j+1}\right)\right]\left(y-y_{j+1}\right)^{2} \\
& +\frac{1+\lambda}{k_{j+1}^{3}}\left[y-y_{j+1}+\frac{k_{j+1}}{h_{i+1}}\left(x-x_{i}\right)\right]^{3} \text {, } \\
& B_{i j}^{2}\left(\Delta_{1}^{2}\right)=\frac{\lambda h_{i}}{\left(h_{i}+h_{i+1}\right) k_{j+2}^{2}\left(k_{j+1}+k_{j+2}\right)}\left[y-y_{j+2}-\frac{k_{j+2}}{h_{i}}\left(x-x_{i}\right)\right]^{3} \text {, } \\
& B_{i j}^{2}\left(\Delta_{2}^{2}\right)=\frac{\lambda}{k_{j+2}^{2}\left(k_{j+1}+k_{j+2}\right)}\left(y-y_{j+2}\right)^{3} \\
& -\frac{\lambda h_{i+1}}{\left(h_{i}+h_{i+1}\right) k_{j+2}^{2}\left(k_{j+1}+k_{j+2}\right)}\left[y-y_{j+2}+\frac{k_{j+2}}{h_{i+1}}\left(x-x_{i}\right)\right]^{3}, \\
& B_{i j}^{2}\left(\Delta_{3}^{2}\right)=-\frac{\lambda}{h_{i}^{2}\left(h_{i}+h_{i+1}\right)}\left(x-x_{i-1}\right)^{3} \text {, } \\
& B_{i j}^{2}\left(\Delta_{4}^{2}\right)=-\frac{\lambda}{h_{i}^{2}\left(h_{i}+h_{i+1}\right)}\left(x-x_{i-1}\right)^{3} \\
& +\frac{\lambda h_{i}}{\left(h_{i}+h_{i+1}\right) k_{j+1}^{2}\left(k_{j+1}+k_{j+2}\right)}\left[y-y_{j+1}+\frac{k_{j+1}}{h_{i}}\left(x-x_{i-1}\right)\right]^{3}, \\
& B_{i j}^{2}\left(\Delta_{5}^{2}\right)=-\frac{\lambda h_{i}}{\left(h_{i}+h_{i+1}\right) k_{j}^{2}\left(k_{j}+k_{j+1}\right)}\left[y-y_{j}+\frac{k_{j}}{h_{i}}\left(x-x_{i-1}\right)\right]^{3} \text {, } \\
& B_{i j}^{2}\left(\Delta_{6}^{2}\right)=-\frac{\lambda}{h_{i}^{2}\left(h_{i}+h_{i+1}\right)}\left(x-x_{i-1}\right)^{3} \\
& -\frac{\lambda h_{i}}{\left(h_{i}+h_{i+1}\right) k_{j+1}^{2}\left(k_{j}+k_{j+1}\right)}\left[y-y_{j}-\frac{k_{j+1}}{h_{i}}\left(x-x_{i-1}\right)\right]^{3} \text {, } \\
& B_{i j}^{2}\left(\Delta_{7}^{2}\right)=-\frac{\lambda}{k_{j}^{2}\left(k_{j}+k_{j+1}\right)}\left(y-y_{j-1}\right)^{3} \text {, } \\
& B_{i j}^{2}\left(\Delta_{8}^{2}\right)=-\frac{\lambda}{k_{j}^{2}\left(k_{j}+k_{j+1}\right)}\left(y-y_{j-1}\right)^{3} \\
& +\frac{\lambda h_{i+1}}{\left(h_{i}+h_{i+1}\right) k_{j}^{2}\left(k_{j}+k_{j+1}\right)}\left[y-y_{j-1}-\frac{k_{j}}{h_{i+1}}\left(x-x_{i}\right)\right]^{3} \text {, }
\end{aligned}
$$




$$
\begin{aligned}
& B_{i j}^{2}\left(\Delta_{9}^{2}\right)=-\frac{\lambda h_{i+2}}{\left(h_{i+1}+h_{i+2}\right) k_{j}^{2}\left(k_{j}+k_{j+1}\right)}\left[y-y_{j-1}-\frac{k_{j}}{h_{i+2}}\left(x-x_{i+1}\right)\right]^{3}, \\
& B_{i j}^{2}\left(\Delta_{10}^{2}\right)=-\frac{\lambda}{k_{j}^{2}\left(k_{j}+k_{j+1}\right)}\left(y-y_{j-1}\right)^{3} \\
& +\frac{\lambda h_{i+1}}{\left(h_{i+1}+h_{i+2}\right) k_{j}^{2}\left(k_{j}+k_{j+1}\right)}\left[y-y_{j-1}+\frac{k_{j}}{h_{i+1}}\left(x-x_{i+1}\right)\right]^{3} \text {, } \\
& B_{i j}^{2}\left(\Delta_{11}^{2}\right)=\frac{\lambda}{h_{i+2}^{2}\left(h_{i+1}+h_{i+2}\right)}\left(x-x_{i+2}\right)^{3}, \\
& B_{i j}^{2}\left(\Delta_{12}^{2}\right)=\frac{\lambda}{h_{i+2}^{2}\left(h_{i+1}+h_{i+2}\right)}\left(x-x_{i+2}\right)^{3} \\
& -\frac{\lambda h_{i+2}}{\left(h_{i+1}+h_{i+2}\right) k_{j+1}^{2}\left(k_{j}+k_{j+1}\right)}\left[y-y_{j}+\frac{k_{j+1}}{h_{i+2}}\left(x-x_{i+2}\right)\right]^{3}, \\
& B_{i j}^{2}\left(\Delta_{13}^{2}\right)=\frac{\lambda h_{i+2}}{\left(h_{i+1}+h_{i+2}\right) k_{j+2}^{2}\left(k_{j+1}+k_{j+2}\right)}\left[y-y_{j+2}+\frac{k_{j+2}}{h_{i+2}}\left(x-x_{i+1}\right)\right]^{3} \text {, } \\
& B_{i j}^{2}\left(\Delta_{14}^{2}\right)=\frac{\lambda}{h_{i+2}^{2}\left(h_{i+1}+h_{i+2}\right)}\left(x-x_{i+2}\right)^{3} \\
& +\frac{\lambda h_{i+2}}{\left(h_{i+1}+h_{i+2}\right) k_{j+1}^{2}\left(k_{j+1}+k_{j+2}\right)}\left[y-y_{j+1}-\frac{k_{j+1}}{h_{i+2}}\left(x-x_{i+2}\right)\right]^{3}, \\
& B_{i j}^{2}\left(\Delta_{15}^{2}\right)=\frac{\lambda}{k_{j+2}^{2}\left(k_{j+1}+k_{j+2}\right)}\left(y-y_{j+2}\right)^{3} \text {, } \\
& B_{i j}^{2}\left(\Delta_{16}^{2}\right)=\frac{\lambda}{k_{j+2}^{2}\left(k_{j+1}+k_{j+2}\right)}\left(y-y_{j+2}\right)^{3} \\
& -\frac{\lambda h_{i+1}}{\left(h_{i+1}+h_{i+2}\right) k_{j+2}^{2}\left(k_{j+1}+k_{j+2}\right)}\left[y-y_{j+2}-\frac{k_{j+2}}{h_{i+1}}\left(x-x_{i+1}\right)\right]^{3} \text {, } \\
& B_{i j}^{2}\left(\Delta_{17}^{2}\right)=-\frac{\lambda}{h_{i}^{2}\left(h_{i}+h_{i+1}\right)}\left(x-x_{i-1}\right)^{3} \\
& +\frac{\lambda h_{i}}{\left(h_{i}+h_{i+1}\right) k_{j+1}^{2}\left(k_{j+1}+k_{j+2}\right)}\left[y-y_{j+1}+\frac{k_{j+1}}{h_{i}}\left(x-x_{i-1}\right)\right]^{3} \\
& -\frac{\lambda h_{i}}{\left(h_{i}+h_{i+1}\right) k_{j+1}^{2}\left(k_{j}+k_{j+1}\right)}\left[y-y_{j+1}-\frac{k_{j+1}}{h_{i}}\left(x-x_{i}\right)\right]^{3} \text {, } \\
& B_{i j}^{2}\left(\Delta_{18}^{2}\right)=-\frac{\lambda}{h_{i}^{2}\left(h_{i}+h_{i+1}\right)}\left(x-x_{i-1}\right)^{3} \\
& +\frac{\lambda h_{i}}{\left(h_{i}+h_{i+1}\right) k_{j+1}^{2}\left(k_{j+1}+k_{j+2}\right)}\left[y-y_{j+1}+\frac{k_{j+1}}{h_{i}}\left(x-x_{i-1}\right)\right]^{3}
\end{aligned}
$$




$$
\begin{aligned}
& -\frac{\lambda h_{i}}{\left(h_{i}+h_{i+1}\right) k_{j+1}^{2}\left(k_{j}+k_{j+1}\right)}\left[y-y_{j+1}-\frac{k_{j+1}}{h_{i}}\left(x-x_{i}\right)\right]^{3} \\
& +\frac{3 \lambda}{h_{i} h_{i+1}}\left(\frac{1}{k_{j}+k_{j+1}}-\frac{1}{k_{j+1}+k_{j+2}}\right)\left(x-x_{i}\right)^{2}\left(y-y_{j+1}\right) \\
& +\frac{\lambda\left(h_{i+1}-h_{i}\right)}{h_{i}^{2} h_{i+1}^{2}}\left(\frac{k_{j}}{k_{j}+k_{j+1}}-\frac{k_{j+1}}{k_{j+1}+k_{j+2}}\right)\left(x-x_{i}\right)^{3} \\
& +\frac{\lambda}{h_{i+1}^{2}\left(h_{i+1}+h_{i+2}\right)}\left(x-x_{i}\right)^{3}+\frac{3 \lambda k_{j+2}}{h_{i} h_{i+1}\left(k_{j+1}+k_{j+2}\right)}\left(x-x_{i}\right)^{2}, \\
& B_{i j}^{2}\left(\Delta_{19}^{2}\right)=\frac{\lambda}{k_{j+2}^{2}\left(k_{j+1}+k_{j+2}\right)}\left(y-y_{j+2}\right)^{3} \\
& -\frac{\lambda h_{i+1}}{\left(h_{i}+h_{i+1}\right) k_{j+2}^{2}\left(k_{j+1}+k_{j+2}\right)}\left[y-y_{j+2}+\frac{k_{j+2}}{h_{i+1}}\left(x-x_{i}\right)\right]^{3} \\
& -\frac{\lambda h_{i+1}}{\left(h_{i+1}+h_{i+2}\right) k_{j+2}^{2}\left(k_{j+1}+k_{j+2}\right)}\left[y-y_{j+1}-\frac{k_{j+2}}{h_{i+1}}\left(x-x_{i}\right)\right]^{3} \\
& +\frac{3 \lambda}{k_{j+1} k_{j+2}}\left(\frac{1}{h_{i}+h_{i+1}}-\frac{1}{h_{i+1}+h_{i+2}}\right)\left(x-x_{i}\right)\left(y-y_{j+1}\right)^{2} \\
& +\frac{\lambda\left(k_{j+1}-k_{j+2}\right)}{k_{j+1}^{2} k_{j+2}^{2}}\left(\frac{h_{i+1}}{h_{i+1}+h_{i+2}}-\frac{h_{i}}{h_{i}+h_{i+1}}\right)\left(y-y_{j+1}\right)^{3} \\
& -\frac{\lambda}{k_{j+1}^{2}\left(k_{j}+k_{j+1}\right)}\left(y-y_{j+1}\right)^{3}+\frac{3 \lambda h_{i}}{\left(h_{i}+h_{i+1}\right) k_{j+1} k_{j+2}}\left(y-y_{j+1}\right)^{2} \text {, } \\
& B_{i j}^{2}\left(\Delta_{20}^{2}\right)=\frac{\lambda}{k_{j+2}^{2}\left(k_{j+1}+k_{j+2}\right)}\left(y-y_{j+2}\right)^{3} \\
& -\frac{\lambda h_{i+1}}{\left(h_{i}+h_{i+1}\right) k_{j+2}^{2}\left(k_{j+1}+k_{j+2}\right)}\left[y-y_{j+2}+\frac{k_{j+2}}{h_{i+1}}\left(x-x_{i}\right)\right]^{3} \\
& -\frac{\lambda h_{i+1}}{\left(h_{i+1}+h_{i+2}\right) k_{j+2}^{2}\left(k_{j+1}+k_{j+2}\right)}\left[y-y_{j+1}-\frac{k_{j+2}}{h_{i+1}}\left(x-x_{i}\right)\right]^{3} \text {, } \\
& B_{i j}^{2}\left(\Delta_{21}^{2}\right)=-\frac{\lambda}{k_{j}^{2}\left(k_{j}+k_{j+1}\right)}\left(y-y_{j-1}\right)^{3} \\
& +\frac{\lambda h_{i+1}}{\left(h_{i+1}+h_{i+2}\right) k_{j}^{2}\left(k_{j}+k_{j+1}\right)}\left[y-y_{j-1}+\frac{k_{j}}{h_{i+1}}\left(x-x_{i+1}\right)\right]^{3} \\
& +\frac{\lambda h_{i+1}}{\left(h_{i}+h_{i+1}\right) k_{j}^{2}\left(k_{j}+k_{j+1}\right)}\left[y-y_{j}-\frac{k_{j}}{h_{i+1}}\left(x-x_{i+1}\right)\right]^{3} \text {, } \\
& B_{i j}^{2}\left(\Delta_{22}^{2}\right)=-\frac{\lambda}{k_{j}^{2}\left(k_{j}+k_{j+1}\right)}\left(y-y_{j-1}\right)^{3}
\end{aligned}
$$




$$
\begin{aligned}
& +\frac{\lambda h_{i+1}}{\left(h_{i}+h_{i+1}\right) k_{j}^{2}\left(k_{j}+k_{j+1}\right)}\left[y-y_{j-1}-\frac{k_{j}}{h_{i+1}}\left(x-x_{i}\right)\right]^{3} \\
& +\frac{\lambda h_{i+1}}{\left(h_{i+1}+h_{i+2}\right) k_{j}^{2}\left(k_{j}+k_{j+1}\right)}\left[y-y_{j}+\frac{k_{j}}{h_{i+1}}\left(x-x_{i}\right)\right]^{3} \\
& +\frac{3 \lambda}{k_{j} k_{j+1}}\left(\frac{1}{h_{i}+h_{i+1}}-\frac{1}{h_{i+1}+h_{i+2}}\right)\left(x-x_{i}\right)\left(y-y_{j}\right)^{2} \\
& +\frac{\lambda\left(k_{j+1}-k_{j}\right)}{k_{j}^{2} k_{j+1}^{2}}\left(\frac{h_{i}}{h_{i}+h_{i+1}}-\frac{h_{i+1}}{h_{i+1}+h_{i+2}}\right)\left(y-y_{j}\right)^{3} \\
& +\frac{\lambda}{k_{j+1}^{2}\left(k_{j+1}+k_{j+2}\right)}\left(y-y_{j}\right)^{3}+\frac{3 \lambda h_{i}}{\left(h_{i}+h_{i+1}\right) k_{j} k_{j+1}}\left(y-y_{j}\right)^{2}, \\
& B_{i j}^{2}\left(\Delta_{23}^{2}\right)=\frac{\lambda}{h_{i+2}^{2}\left(h_{i+1}+h_{i+2}\right)}\left(x-x_{i+2}\right)^{3} \\
& +\frac{\lambda h_{i+2}}{\left(h_{i+1}+h_{i+2}\right) k_{j+1}^{2}\left(k_{j+1}+k_{j+2}\right)}\left[y-y_{j+1}-\frac{k_{j+1}}{h_{i+2}}\left(x-x_{i+2}\right)\right]^{3} \\
& -\frac{\lambda h_{i+2}}{\left(h_{i+1}+h_{i+2}\right) k_{j+1}^{2}\left(k_{j}+k_{j+1}\right)}\left[y-y_{j+1}+\frac{k_{j+1}}{h_{i+2}}\left(x-x_{i+1}\right)\right]^{3} \text {, } \\
& B_{i j}^{2}\left(\Delta_{24}^{2}\right)=\frac{\lambda}{h_{i+2}^{2}\left(h_{i+1}+h_{i+2}\right)}\left(x-x_{i+2}\right)^{3} \\
& +\frac{\lambda h_{i+2}}{\left(h_{i+1}+h_{i+2}\right) k_{j+1}^{2}\left(k_{j+1}+k_{j+2}\right)}\left[y-y_{j+1}-\frac{k_{j+1}}{h_{i+2}}\left(x-x_{i+2}\right)\right]^{3} \\
& -\frac{\lambda h_{i+2}}{\left(h_{i+1}+h_{i+2}\right) k_{j+1}^{2}\left(k_{j}+k_{j+1}\right)}\left[y-y_{j+1}+\frac{k_{j+1}}{h_{i+2}}\left(x-x_{i+1}\right)\right]^{3} \\
& +\frac{3 \lambda}{h_{i+1} h_{i+2}}\left(\frac{1}{k_{j}+k_{j+1}}-\frac{1}{k_{j+1}+k_{j+2}}\right)\left(x-x_{i+1}\right)^{2}\left(y-y_{j+1}\right) \\
& +\frac{\lambda\left(h_{i+1}-h_{i+2}\right)}{h_{i+1}^{2} h_{i+2}^{2}}\left(\frac{k_{j+1}}{k_{j+1}+k_{j+2}}-\frac{k_{j}}{k_{j}+k_{j+1}}\right)\left(x-x_{i+1}\right)^{3} \\
& -\frac{\lambda}{h_{i+1}^{2}\left(h_{i}+h_{i+1}\right)}\left(x-x_{i+1}\right)^{3}+\frac{3 \lambda k_{j+2}}{h_{i+1} h_{i+2}\left(k_{j+1}+k_{j+2}\right)}\left(x-x_{i+1}\right)^{2} \text {. }
\end{aligned}
$$

\section{References}

[1] C. Dagnino and P. Lamberti, On the approximation power of bivariate quadratic $C^{1}$ splines, J. Comput. Appl. Math., 131 (2001), pp. 321-332.

[2] C. Dagnino and P. LAmberTI, Some performances of local bivariate quadratic $C^{1}$ quasiinterpolating splines on nonuniform type-2 triangulations, J. Comput. Appl. Math., 173 (2005), pp. 21-37. 
[3] G. Farin, Curves and Surfaces for Computer Aided Geometric Design, A Practical Guide, Academic Press, Boston, 1992.

[4] C. J. Li AND J. Chen, On the dimensions of bivariate spline spaces and their stability, J. Comput. Appl. Math., 236 (2011), pp. 765-774.

[5] C. J. Li AND R. H. WANG,, Bivariate cubic spline space and bivariate cubic NURBS surfaces, Proceedings of Geometric Modeling and Processing 2004, IEEE Computer Society Pressvol, pp. $115-123$.

[6] H. W. Liu, D. Hong and D. Q. Cao, Bivariate $C^{1}$ cubic spline space over a nonuniform type-2 triangulation and its subspaces with boundary conditions, J. Comput. Math. Appl., 49 (2005), pp. 1853-1865.

[7] L. Piegl ANd W. TilleR,, The NURBS Book, Spring-Verlag, Berlin, 1997.

[8] J. Qian AND H. Y. Liu, Relationship between UAH B splines and uniform B splines, J. Hohai Univ., (Natural Sciences), 34(5) (2006), pp. 599-602.

[9] J. QIAN AND Y. H. TANG, On non-uniform Algebraic-Hyperbolic (NUAH) B-splines, Numer. Math. A Journal of Chinese Universities, 15(4) (2006), pp. 320-335.

[10] J. QIAN AND Y. H. TANG, The application of H Bezier-like curves in engineering, J. Numer. Methods Comput. Appl., 28(3) (2007), pp. 167-178.

[11] J. QIAN, R. H. WANG AND C. J. LI, The bases of non-uniform cubic spline space $S_{3}^{1,2}\left(\Delta_{m n}^{(2)}\right)$, Numer. Math. Theor. Meth. Appl., 5(4) (2012), pp. 635-652.

[12] J. QIAN, R. H. WANG, C. G. ZHU AND F. WANG, On spline quasi-interpolation in cubic spline space $S_{3}^{1,2}\left(\Delta_{m n}^{(2)}\right)$, Submitted.

[13] L. L. SCHUMAKER, On the dimension of spaces of piecewise polynomials in two variables, Multivariate Approximation Theory, W. Schempp and K. Zeller (Eds.), Birkhauser, Basel, 1979, pp. $396-412$.

[14] L. L. Schumaker, Spline Functions: Basic Theory, Krieger Publishing Company, Malabar FL, 1993.

[15] G. Z. WAng, Q. Y. Chen And M. H. Zhou, NUAT B-spline Curves, Comput. Aided Geom. Design, 21 (2004), pp. 193-205.

[16] G. J Wang, G. Z. Wang and J. M. Zheng, Computer Aided Geometric Design, China Higher Education Press/Spring-Verlag Berlin, Beijing/Heidelberg, 2001.

[17] R. H. WANG, The structural characterization and interpolation for multivariate splines, Acta Math. Sinica, 18 (1975), pp. 91-106.

[18] R. H. WAng, X. Q. Shi, Z. X. Luo And Z. X. Su, Multivariate Spline Functions and Their Applications, Science Press/Kluwer Academic Publishers, Beijing, New York, Dordrecht, Boston, London, 2001.

[19] R. H. WANG AND C. J. LI, Bivariate quartic spline spaces and quasi-interpolation operators, J. Comput. Appl. Math., 190 (2006), pp. 325-338.

[20] R. H. WANG AND Y. LU, Quasi-interpolating operators and their applications in hypersingular integrals, J. Comput. Math., 16(4) (1998), pp. 337-344.

[21] R. H. WANG AND Y. Lu, Quasi-interpolating operators in $S_{2}^{1}\left(\Delta_{m n}^{2} *\right)$ on non-uniform type-2 triangulations, Numer. Math. A Journal of Chinese Universities, 2 (1999), pp. 97-103.

[22] S. M. WANG, Spline interpolation over type-2 triangulations, Appl. Math. Comput., 49 (1992), pp. 299-313.

[23] S. M. WANG AND C. L. WANG, Smooth interpolation on some triangulations, Utilitas Math., 41 (1992), pp. 309-317.

[24] Z. Q. XU AND R. H. WANG, The instability degree in the dimension of spaces of bivariate spline, Approx. Theory Appl., 18(1) (2002), pp. 68-80. 